\title{
SINGULARITY AND FINE FRACTAL PROPERTIES OF A CERTAIN CLASS OF INFINITE BERNOULLI CONVOLUTIONS WITH AN ESSENTIAL INTERSECTION
}

UDC 519.21

\author{
M. V. LEBID' AND G. M. TORBIN
}

ABstract. We prove that the probability distribution of a random variable $\xi$ represented in the form of an infinite series

$$
\xi=\sum_{k=1}^{\infty} \xi_{k} a_{k}
$$

is singular, where $\xi_{k}$ are independent Bernoulli random variables and where the sequence $\left\{a_{k}\right\}$ is such that the series $\sum_{k=1}^{\infty} a_{k}$ converges, $a_{k} \geq 0$ for all $k \geq 1$, and, for an arbitrary $k \in \mathbf{N}$, there exists $s_{k} \in \mathbf{N} \cup\{0\}$ for which $s_{k}>0$ for infinitely many indices $k$ and $a_{k}=a_{k+1}=\cdots=a_{k+s_{k}} \geq r_{k+s_{k}}$, where $r_{k}$ is the tail of the series, namely

$$
r_{k}=\sum_{i=k+1}^{\infty} a_{i} .
$$

Under these assumptions, it is shown that the corresponding distribution is a Bernoulli convolution with essential intersections (that is, almost all with respect to the Hausdorff-Besicovitch dimension points of the spectrum have continuum many different expansions of the form $\sum_{k=1}^{\infty} \omega_{k} a_{k}$, where $\left.\omega_{k} \in\{0,1\}\right)$. Our main attention is paid to the studies of fractal properties of singularly continuous probability measures $\mu_{\xi}$. In particular, fractal properties of the spectra (minimal closed supports of the above measures) and minimal in the sense of the Hausdorff-Besicovitch dimension dimensional supports of such probability distributions are studied in detail.

\section{INTRODUCTION}

Let $\mu_{\xi}=\mu$ be the distribution of the random variable

$$
\xi=\sum_{k=1}^{\infty} \xi_{k} a_{k},
$$

where $\sum_{k=1}^{\infty} a_{k}$ is a series whose terms are nonnegative and where $\xi_{k}$ are independent random variables assuming two values 0 and 1 with probabilities $p_{0 k}$ and $p_{1 k}$, respectively. The distribution $\mu_{\xi}$ is called an infinite Bernoulli convolution.

It is shown in the paper [1] that, when studying the Lebesgue structure and the fractal properties of the measure $\mu_{\xi}$, one can restrict consideration without loss of generality to the case where the matrix $\left\|p_{i k}\right\|$ does not contain zeros (that is, $p_{0 k} \in(0,1)$ for all

2010 Mathematics Subject Classification. Primary 60G30, 11K55, 28A80.

Key words and phrases. Infinite Bernoulli convolutions, fractals, singularly continuous probability measures, Hausdorff-Besicovitch dimension of a set, Hausdorff dimension of a measure, faithful covering systems.

The research of the first named author was partially supported by the DFG Grant 436 113/97.

The research of the second named author was partially supported by the DFG Grants 436 UKR 113/97, DFG KO 1989/6-1, and Humboldt Foundation. 
$k \in \mathbf{N}$ ) and where the sequence $\left\{a_{k}\right\}$ is nondecreasing (that is, $a_{k} \geq a_{k+1}$ for all $k \in \mathbf{N}$ ) and such that $\sum_{k=1}^{\infty} a_{k}=1$.

A theorem due to Jessen and Wintner [12] says that the distribution of $\xi$ is pure (in other words, $\mu_{\xi}$ is either purely discrete, or purely absolutely continuous with respect to the Lebesgue measure, or purely singularly continuous). A theorem due to Lévy [13. provides necessary and sufficient conditions for $\mu$ to be purely discrete, namely the measure $\mu_{\xi}$ is discrete if and only if

$$
M=\prod_{k=1}^{\infty} \max \left\{p_{0 k}, p_{1 k}\right\}>0 .
$$

The criteria for $\mu_{\xi}$ to be purely absolutely continuous with respect to the Lebesgue measure (or purely singular) are not known yet even in the case of random power series, despite the problem having been actively studied over last 80 years or so (see, for example, [3, 8, 10, 15, 16, 18]). Surveys of problems and possible solutions in this field are given in [11, 14. Some applications of infinite Bernoulli convolutions are discussed in [5, 14]. If the series $\sum_{k=1}^{\infty} a_{k}$ converges "fast enough", that is, if

$$
a_{k} \geq r_{k}:=\sum_{n=k+1}^{\infty} a_{n}
$$

for all sufficiently large $k$, then the Lebesgue structure and fractal properties of Bernoulli convolutions are studied rather well (see [1, 7, 17]). In contrast, if the inequality $a_{k}<r_{k}$ occurs for an infinite number of indices $k$, then these problems are studied much less. The main problem in this case is how to obtain appropriate properties of the Bernoulli convolutions for which almost all (with respect to the Lebesgue measure or in the sense of the Hausdorff-Besicovitch dimension) points of the spectrum have continuum many different expansions of the form $\sum_{k=1}^{\infty} \omega_{k} a_{k}$, where $\omega_{k} \in\{0,1\}$. The probability measures of this type belong to the class of the so-called Bernoulli convolutions with essential intersections (see [11]).

This paper is devoted to the study of measures with essential intersections. The main aim of the paper is to prove the singularity of the distribution of the random variable $\xi$ and to investigate its fine fractal properties for the case where the random variables $\xi_{k}$ are identically distributed and the sequence $\left\{a_{k}\right\}$ is such that

$$
\forall k \in \mathbf{N}, \exists s_{k} \in N_{0}:=\mathbf{N} \cup\{0\}: \quad a_{k}=a_{k+1}=\cdots=a_{k+s_{k}} \geq r_{k+s_{k}},
$$

and moreover $s_{k}>0$ for an infinite number of indices $k$.

\section{Singularity of the Distribution of $\xi$}

Let $\Omega=\{0,1\}^{\infty}$. For a fixed series $\sum_{k=1}^{\infty} a_{k}$, consider the mapping $\varphi: \Omega \rightarrow \mathbf{R}$ defined as follows:

$$
\forall \omega=\left(\omega_{1}, \omega_{2}, \ldots, \omega_{k}, \ldots\right) \in \Omega: \quad \varphi(\omega)=\sum_{k=1}^{\infty} \omega_{k} a_{k} .
$$

The set $\Delta^{\prime}:=\varphi(\Omega)=\{x: \exists \omega \in \Omega \wedge \varphi(\omega)=x\}$ is called the set of incomplete sums of the series $\sum_{k=1}^{\infty} a_{k}$. Since $p_{i k}>0$ for all $i \in\{0,1\}$ and $k \in \mathbf{N}$, the set $\Delta^{\prime}$ is the spectrum (in other words, the minimal closed support) of the distribution of the random variable $\xi$.

Following paper [1], the set of real numbers of the form

$$
\sum_{n=1}^{m} c_{n} a_{n}+\sum_{n=m+1}^{\infty} \omega_{n} a_{n}
$$


where $\omega_{n} \in\{0,1\}$, is called the cylinder of rank $m$ with the base $c_{1}, \ldots, c_{m}, c_{i} \in\{0,1\}$. It is clear that the set $\Delta_{c_{1}, \ldots, c_{m}}^{\prime}$ is the image under the mapping $\varphi$ of the cylinder belonging to $\Omega$ whose base is $c_{1}, \ldots, c_{m}\left(c_{i} \in\{0,1\}\right)$.

The interval

$$
\Delta_{c_{1}, \ldots, c_{m}}=\left[\sum_{n=1}^{m} c_{n} a_{n}, r_{m}+\sum_{n=1}^{m} c_{n} a_{n}\right]
$$

is called the cylindrical interval of rank $m$ with the base $c_{1}, \ldots, c_{m}$. Note that

$$
\Delta_{c_{1}, \ldots, c_{m}}^{\prime} \subset \Delta_{c_{1}, \ldots, c_{m}} .
$$

We introduce some auxiliary notation. Let $\left\{k_{n}\right\}$ be a sequence of nonnegative integer numbers such that $i \in\left\{k_{n}\right\}$ if and only if $s_{i}=0$. Also let $l_{n}=k_{n}-k_{n-1}, k_{0}=0$.

Some general properties of cylinders and cylindrical intervals follow directly from their definitions (note that the sequence $\left\{a_{k}\right\}$ is arbitrary), namely

1) $\inf \Delta_{c_{1}, \ldots, c_{m}}=\inf \Delta_{c_{1}, \ldots, c_{m}}^{\prime} ; \sup \Delta_{c_{1}, \ldots, c_{m}}=\sup \Delta_{c_{1}, \ldots, c_{m}}^{\prime}$

2) $\Delta_{c_{1}, \ldots, c_{m}}^{\prime}=\Delta_{c_{1}, \ldots, c_{m} 0}^{\prime} \cup \Delta_{c_{1}, \ldots, c_{m} 1}^{\prime}$;

3) inf $\Delta_{c_{1}, \ldots, c_{m}}=\inf \Delta_{c_{1}, \ldots, c_{m} 0}, \sup \Delta_{c_{1}, \ldots, c_{m}}=\sup \Delta_{c_{1}, \ldots, c_{m} 1}$;

4) $\left|\Delta_{c_{1}, \ldots, c_{m}}\right|=r_{m} \rightarrow 0, m \rightarrow \infty$;

5) $\bigcap_{m=1}^{\infty} \Delta_{c_{1}, \ldots, c_{m}}=\bigcap_{m=1}^{\infty} \Delta_{c_{1}, \ldots, c_{m}}^{\prime} \equiv \Delta_{c_{1}, \ldots, c_{m}}=x \in \Delta^{\prime} \subset[0,1]$.

The following property is a consequence of condition (3):

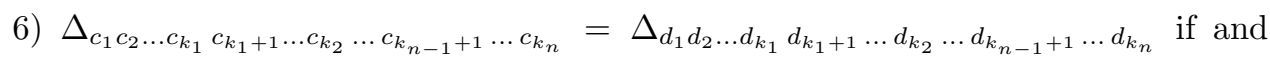
only if

$$
\left\{\begin{array}{l}
\sum_{i=1}^{k_{1}} c_{i}=\sum_{i=1}^{k_{1}} d_{i}, \\
\sum_{i=k_{1}+1}^{k_{2}} c_{i}=\sum_{i=k_{1}+1}^{k_{2}} d_{i}, \\
\ldots \ldots \ldots \ldots \ldots \ldots \ldots \ldots \ldots \ldots \ldots \ldots \\
\sum_{i=k_{n-1}+1}^{k_{n}} c_{i}=\sum_{i=k_{n-1}+1}^{k_{n}} d_{i} .
\end{array}\right.
$$

Below is the description of those points of the spectrum that have only a finite number of representations. Let

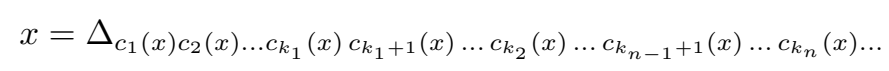

be one of the representations of a point $x$. If $\sum_{i=k_{n-1}+1}^{k_{n}} c_{i}(x) \notin\left\{0, l_{n}\right\}$ for an infinite number of indices $n$, then the point $x$ has continuum many representations in the form $\sum_{i=1}^{\infty} c_{i} a_{i}$. Indeed, property 6 ) implies that if

$$
\sum_{i=k_{n-1}+1}^{k_{n}} c_{i}(x) \notin\left\{0, l_{n}\right\},
$$

then the equation

$$
x_{1}+x_{2}+\cdots+x_{l_{n}}=\sum_{i=k_{n-1}+1}^{k_{n}} c_{i}(x)
$$

has at least two different solutions $\left(x_{1}^{(1)}, x_{2}^{(1)}, \ldots, x_{l_{n}}^{(1)}\right)$ and $\left(x_{1}^{(2)}, x_{2}^{(2)}, \ldots, x_{l_{n}}^{(2)}\right)$, where $x_{i}^{(j)} \in\{0,1\}$ for all $i=1, \ldots, l_{n}$ and $j=1,2$. Applying this reasoning to those numbers 
$n$ for which

$$
\sum_{i=k_{n-1}+1}^{k_{n}} c_{i}(x) \in\left\{1,2, \ldots, l_{n}-1\right\}
$$

and taking into account that $l_{n}>1$ for infinitely many indices $n$, we prove the result desired.

Note also that there are points belonging to the spectrum that have a unique representation in the form of (5). If $a_{k_{n}}>r_{k_{n}}$ for infinitely many indices $n$, then all points of the form

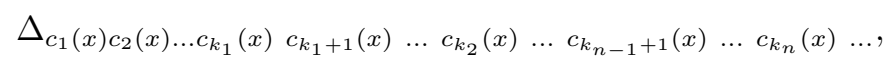

where

$$
c_{k_{n-1}+1}(x)+c_{k_{n-1}+2}+\cdots+c_{k_{n}} \in\left\{0, l_{n}\right\}, \quad \forall n \in \mathbf{N},
$$

have a unique representation in the form of (5). If $a_{k_{n}}=r_{k_{n}}$ for an infinite number of indices $n$, then some points of the form (6) have exactly two different representations (note that the set of such points is countable): one of these representations has " 0 " in period, while the other one has " 1 " in period. Thus the set of those points that have a unique representation in the form of (5) is uncountable.

It is clear that if

$$
c_{k_{n-1}+1}(x)+c_{k_{n-1}+2}(x)+\cdots+c_{k_{n}}(x) \in\left\{0, l_{n}\right\}
$$

for all sufficiently large $n \in \mathbf{N}$, then the point $x$ has a finite number of representations in the form of (5). In Section 3 , we determine the dimension of the set of those points for which there exist continuum many different representations. We also determine the dimension of the set of points that have a finite number of representations.

Our current goal is to show that the distribution of the random variable $\xi$ is a probability measure with independent $\widetilde{Q}$-symbols (see [2]). Having this goal in mind, we introduce the sequence $\left\{m_{n}\right\}$ by

$$
m_{n}= \begin{cases}l_{n}+1, & \text { if } a_{k_{n}}=r_{k_{n}}, \\ 2 l_{n}+1, & \text { if } a_{k_{n}}>r_{k_{n}} .\end{cases}
$$

For every $n$, define the stochastic vector column

$$
\vec{q}_{n}=\left(q_{0 n}, q_{1 n}, \ldots, q_{m_{n}-1, n}\right)
$$

as follows:

1) If $m_{n}=l_{n}+1$, then

$$
q_{i n}=\frac{1}{l_{n}+1}, \quad i \in\left\{0,1,2, \ldots, m_{n}-1\right\}=B_{n} .
$$

2) If $m_{n}=2 l_{n}+1$, then

$$
\begin{gathered}
q_{i n}=\frac{r_{k_{n}}}{r_{k_{n-1}}}, \quad i \in\left\{0,2,4, \ldots, m_{n}-1\right\}=B_{n}, \\
q_{\text {in }}=\frac{a_{k_{n}}-r_{k_{n}}}{r_{k_{n-1}}}, \quad i \in\left\{1,3,5, \ldots, m_{n}-2\right\} .
\end{gathered}
$$

The stochastic matrix $\widetilde{Q}=\left\|q_{i n}\right\|$, whose column $n$ coincides with the stochastic vector $\vec{q}_{n}$, generates a $\widetilde{Q}$-representation of numbers of the interval $[0,1]$ in the following way. Let $A_{n}=\left\{0,1, \ldots, m_{n}-1\right\}$ and $\gamma_{n} \in A_{n}$. Consider the mapping

$$
f: A_{1} \times A_{2} \times \cdots \times A_{n} \times \cdots \mapsto[0,1]
$$


given by

$$
f\left(\left\{\gamma_{n}\right\}\right)=x=\beta_{\gamma_{1} 1}+\sum_{n=2}^{\infty} \beta_{\gamma_{n} n} \prod_{i=1}^{n-1} q_{\gamma_{i} i},
$$

where $\beta_{\gamma_{n} n}=\sum_{j=0}^{\gamma_{n}-1} q_{j n}$. We also write

$$
x=\tilde{\Delta}_{\gamma_{1} \gamma_{2} \ldots \gamma_{n} \ldots,}, \quad \gamma_{n} \in A_{n} .
$$

The latter expression is called the $\widetilde{Q}$-representation of a number $x$ (see [2, 18, for details).

The interval

$$
\tilde{\Delta}_{\gamma_{1} \ldots \gamma_{m}}=\left[\beta_{\gamma_{1} 1}+\sum_{n=2}^{m} \beta_{\gamma_{n} n} \prod_{i=1}^{n-1} q_{\gamma_{i} i}, \beta_{\gamma_{1} 1}+\sum_{n=2}^{m} \beta_{\gamma_{n} n} \prod_{i=1}^{n-1} q_{\gamma_{i} i}+\prod_{i=1}^{m} q_{\gamma_{i} i}\right]
$$

is called the cylindrical interval of rank $m$ with the base $\gamma_{1} \ldots \gamma_{m}$ for the $\widetilde{Q}$-representation of a number $x$. Since the cylinders $\Delta_{c_{1} \ldots c_{k_{n}}}^{\prime}$ of rank $k_{n}$ are either disjoint or coincide, there exists a correspondence between the set of cylindrical intervals $\Delta_{c_{1} c_{2} \ldots c_{k_{n}}}$ of rank $k_{n}$ and the set of $\widetilde{Q}$-cylinders $\tilde{\Delta}_{\gamma_{1} \gamma_{2} \ldots \gamma_{n}}, \gamma_{i} \in B_{i}$. The correspondence mentioned above is generated by the mapping

$$
\gamma_{i}= \begin{cases}c_{k_{i-1}+1}+\cdots+c_{k_{i}}, & \text { if } a_{k_{i}}=r_{k_{i}} \\ 2\left(c_{k_{i-1}+1}+\cdots+c_{k_{i}}\right), & \text { if } a_{k_{i}}>r_{k_{i}}\end{cases}
$$

This means, for a fixed series $\sum_{k=1}^{\infty} a_{k}$ and for an arbitrary collection

$$
c_{1} c_{2} \ldots c_{k_{1}} c_{k_{1}+1} \ldots c_{k_{2}} \ldots c_{k_{n-1}+1} \ldots c_{k_{n}}, \quad c_{i} \in\{0,1\}
$$

that there exists a unique set $\gamma_{1}, \gamma_{2}, \ldots, \gamma_{n}\left(\gamma_{i} \in B_{i}\right)$ such that

$$
\Delta_{c_{1} c_{2} \ldots c_{k_{n}}}=\tilde{\Delta}_{\gamma_{1} \gamma_{2} \ldots \gamma_{n}}
$$

where $\gamma_{i}$ is defined by $l_{i}$ symbols $c_{k_{i-1}+1} \ldots c_{k_{i}}$ according to the condition $a_{k_{n}}=r_{k_{n}}$.

Let $\left\{\tilde{\xi}_{n}\right\}$ be a sequence of independent random variables assuming the values

$$
0,1, \ldots, m_{n}-1
$$

with probabilities $\tilde{p}_{0 n}, \tilde{p}_{1 n}, \ldots, \tilde{p}_{m_{n}-1, n}$, respectively, where

$$
\tilde{p}_{i n}=C_{l_{n}}^{i} q^{l_{n}-i} p^{i}
$$

for $a_{k_{n}}=r_{k_{n}}$, and let

$$
\tilde{p}_{i n}= \begin{cases}C_{l_{n}}^{i / 2} q^{l_{n}-i / 2} p^{i / 2}, & \text { if } i \text { is even, } \\ 0, & \text { if } i \text { is odd }\end{cases}
$$

for $a_{k_{n}}>r_{k_{n}}$.

The random variable $\tilde{\xi}$ with independent $\widetilde{Q}$-symbols $\tilde{\xi}_{n}$,

$$
\tilde{\xi}=f\left(\tilde{\xi}_{1}, \tilde{\xi}_{2}, \ldots, \tilde{\xi}_{n}, \ldots\right)=\beta_{\tilde{\xi}_{1} 1}+\sum_{n=2}^{\infty} \beta_{\tilde{\xi}_{n} n} \prod_{i=1}^{n-1} q_{\tilde{\xi}_{i} i}=: \tilde{\Delta}_{\tilde{\xi}_{1}} \tilde{\xi}_{2} \ldots \tilde{\xi}_{n} \ldots,
$$

is determined by the stochastic matrix $\left\|q_{i n}\right\|$ and the sequence of independent random variables $\left\{\tilde{\xi}_{n}\right\}$.

Remark 2.1. The random variables $\xi$ and $\tilde{\xi}$ are identically distributed. Indeed, it is sufficient to show that $\mathrm{P}_{\xi}\left(\Delta_{c_{1} c_{2} \ldots c_{k_{n}}}\right)=\mathrm{P}_{\tilde{\xi}}\left(\tilde{\Delta}_{\gamma_{1} \gamma_{2} \ldots \gamma_{n}}\right)$ for all $n \in \mathbf{N}$, where

$$
\gamma_{i}= \begin{cases}c_{k_{i-1}+1}+\cdots+c_{k_{i}}, & \text { if } a_{k_{i}}=r_{k_{i}}, \\ 2\left(c_{k_{i-1}+1}+\cdots+c_{k_{i}}\right), & \text { if } a_{k_{i}}>r_{k_{i}} .\end{cases}
$$


This equality is obvious in view of the construction of the random variable $\tilde{\xi}$ and in view of the properties of the binomial distribution, since the random variables $\xi_{1}, \xi_{2}, \ldots, \xi_{k_{n}}$ are independent and identically distributed.

Lemma 2.1. The Lebesgue measure of the spectrum of the random variable $\xi$ is equal to

$$
\lim _{n \rightarrow \infty} r_{k_{n}}\left(\prod_{j=1}^{n}\left(l_{j}+1\right)\right)
$$

Proof. The spectrum of the random variable $\tilde{\xi}$ is an infinite intersection of unions of $\widetilde{Q}$ cylindrical intervals (each being of a nonzero measure $\mu_{\xi}$ ) of all ranks. Such $\widetilde{Q}$-cylindrical intervals of rank $n$ coincide, and the total number of these cylindrical intervals is equal to $l_{n}+1$. Hence

$$
S_{\tilde{\xi}}=\bigcap_{n=1}^{\infty} \bigcup_{\gamma_{1} \in B_{1}} \cdots \bigcup_{\gamma_{n-1} \in B_{n-1}} \bigcup_{\gamma_{n} \in B_{n}} \tilde{\Delta}_{\gamma_{1} \gamma_{2} \ldots \gamma_{n-1} \gamma_{n}} .
$$

Since $\mu_{\tilde{\xi}}\left(\tilde{\Delta}_{\gamma_{1} \gamma_{2} \ldots \gamma_{n-1} \gamma_{n}}\right)=\widetilde{p}_{\gamma_{1} 1} \widetilde{p}_{\gamma_{2} 2} \ldots \widetilde{p}_{\gamma_{n} n}>0$ and $\lambda\left(\tilde{\Delta}_{\gamma_{1} \gamma_{2} \ldots \gamma_{n-1} \gamma_{n}}\right)=r_{k_{n}}$, the continuity of the Lebesgue measure implies that

$$
\lambda\left(S_{\bar{\xi}}\right)=\lambda\left(\bigcap_{n=1}^{\infty} \bigcup_{\gamma_{1} \in B_{1}} \ldots \bigcup_{\gamma_{n-1} \in B_{n-1}} \bigcup_{\gamma_{n} \in B_{n}} \tilde{\Delta}_{\gamma_{1} \gamma_{2} \ldots \gamma_{n-1} \gamma_{n}}\right)=\lim _{n \rightarrow \infty} r_{k_{n}} \prod_{j=1}^{n}\left(l_{j}+1\right),
$$

and this completes the proof of Lemma 2.1 .

Lemma 2.2. Let $p=1-q \in[0,1]$. Then

$$
\sqrt{\frac{1}{n+1}} \sum_{i=0}^{n} \sqrt{C_{n}^{i} q^{n-i} p^{i}} \rightarrow 0, \quad n \rightarrow \infty .
$$

Proof. Let $\varepsilon>0$ and let and $S_{n}$ denote the total number of "successes" in a series of $n$ independent Bernoulli trials, where a "success" occurs with probability $p$. By the law of large numbers,

$$
\lim _{n \rightarrow \infty} \mathrm{P}\left\{\left|\frac{S_{n}}{n}-p\right|>\varepsilon\right\}=0 .
$$

Thus there exists $n_{0} \in \mathbf{N}$ such that

$$
\mathrm{P}\left\{\left|\frac{S_{n}}{n}-p\right|>\varepsilon\right\}<\varepsilon
$$

for all $n>n_{0}$. The Cauchy-Bunyakovskiǔ inequality,

$$
\left(1 \cdot a_{1}+\cdots+1 \cdot a_{m}\right)^{2} \leq m \cdot\left(a_{1}^{2}+\cdots+a_{m}^{2}\right),
$$

together with (8) implies that

$$
\begin{aligned}
\sum_{i:\left|\frac{i}{n}-p\right|>\varepsilon}^{n} \sqrt{C_{n}^{i} q^{n-i} p^{i}} & \leq \sqrt{n+1} \sqrt{\left(\sum_{i:\left|\frac{i}{n}-p\right|>\varepsilon}^{n} C_{n}^{i} q^{n-i} p^{i}\right)} \\
& =\sqrt{n+1} \sqrt{\mathrm{P}\left\{\left|\frac{S_{n}}{n}-p\right|>\varepsilon\right\}} \leq \sqrt{\varepsilon(n+1)}
\end{aligned}
$$

if $\left|\frac{i}{n}-p\right|>\varepsilon$. 
Since there exist at most $2 n \varepsilon+1$ positive integer numbers $i$ such that $\left|\frac{i}{n}-p\right| \leq \varepsilon$, we again use the Cauchy-Bunyakovskiı inequality and the preceding result to prove that

$$
\sum_{i:\left|\frac{i}{n}-p\right| \leq \varepsilon}^{n} \sqrt{C_{n}^{i} q^{n-i} p^{i}} \leq \sqrt{2 \varepsilon n+1} \sqrt{\left(\sum_{i:\left|\frac{i}{n}-p\right| \leq \varepsilon}^{n} C_{n}^{i} q^{n-i} p^{i}\right)} \leq \sqrt{2 \varepsilon n+1} .
$$

Inequalities (9) and (10) imply that

$$
\begin{aligned}
\sum_{i=0}^{n} \sqrt{C_{n}^{i} q^{n-i} p^{i}} & =\sum_{i:\left|\frac{i}{n}-p\right| \leq \varepsilon}^{n} \sqrt{C_{n}^{i} q^{n-i} p^{i}}+\sum_{i:\left|\frac{i}{n}-p\right|>\varepsilon}^{n} \sqrt{C_{n}^{i} q^{n-i} p^{i}} \\
& \leq \sqrt{\varepsilon} \sqrt{n+1}+\sqrt{2 \varepsilon n+1}<3 \sqrt{\varepsilon} \sqrt{n+1}+1
\end{aligned}
$$

for $n>n_{0}$.

Therefore

$$
\sqrt{\frac{1}{n+1}} \sum_{i=0}^{n} \sqrt{C_{n}^{i} q^{n-i} p^{i}} \leq 3 \sqrt{\varepsilon}+\sqrt{\frac{1}{n+1}}
$$

which completes the proof Lemma 2.2 ,

Corollary 2.1. Let $p=1-q \in[0,1]$ and let $K_{0}$ be an arbitrary number of $(0,1)$. Then there exists $n_{0}=n_{0}\left(p, K_{0}\right) \in \mathbf{N}$ such that

$$
\sqrt{\frac{1}{n+1}} \sum_{i=0}^{n} \sqrt{C_{n}^{i} q^{n-i} p^{i}} \leq K_{0}<1, \quad \forall n>n_{0} .
$$

Consider the expression

$$
\sqrt{\frac{1}{m+1}} \sum_{i=0}^{m} \sqrt{C_{m}^{i} q^{m-i} p^{i}}
$$

as a function of $v(p)$ that depends on $p=1-q \in[0,1]$.

Lemma 2.3. Let $m \in \mathbf{N} \backslash\{1\}$. Then

$$
v(p)=\sqrt{\frac{1}{m+1}} \sum_{i=0}^{m} \sqrt{C_{m}^{i} q^{m-i} p^{i}} \leq K_{m}<1, \quad \forall p=1-q \in[0,1],
$$

where $K_{m}$ is a constant that depends on $m$.

Proof. Consider the function

$$
\varphi\left(x_{0}, x_{1}, \ldots, x_{m}\right)=\sqrt{x_{0}}+\sqrt{x_{1}}+\cdots+\sqrt{x_{m}}
$$

in the domain $G$ of the hyperplane $x_{0}+x_{1}+\cdots+x_{m}=1$ that belongs to the $(m+1)$ dimensional cube $[0,1]^{m+1}$. Since

$$
\overrightarrow{\left(\sqrt{x_{0}}, \sqrt{x_{1}}, \ldots, \sqrt{x_{m}}\right)} \overrightarrow{(1,1, \ldots, 1)} \leq\left|\overrightarrow{\left(\sqrt{x_{0}}, \sqrt{x_{1}}, \ldots, \sqrt{x_{m}}\right)}\right||\overrightarrow{(1,1, \ldots, 1)}|=\sqrt{m+1},
$$

the function $\varphi\left(x_{0}, x_{1}, \ldots, x_{m}\right)$ (being continuous in the domain $G$ ) attains its maximal value $\sqrt{m+1}$ at the point with equal coordinates

$$
x_{0}=x_{1}=\cdots=x_{m}=\frac{1}{m+1}
$$

and

$$
\varphi\left(x_{0}, x_{1}, \ldots, x_{m}\right)<\sqrt{m+1}
$$

for all other points of the set $G$. 
Let

$$
v(p)=\sqrt{\frac{1}{m+1}} \sum_{i=0}^{m} \sqrt{C_{m}^{i} q^{m-i} p^{i}},
$$

where $p=1-q \in[0,1]$. This function is continuous in $[0,1]$ and is bounded from above:

$$
v(p) \leq \max \left(\frac{\varphi\left(x_{0}, x_{1}, \ldots, x_{m}\right)}{\sqrt{m+1}}\right)=1
$$

The inequality becomes an equality only for the case of

$$
C_{m}^{i} q^{m-i} p^{i}=\frac{1}{m+1}, \quad \forall i=0, \ldots, m .
$$

If $m \geq 2$, then either $p^{m}$ or $q^{m}$ is less than $\frac{1}{m+1}$. Hence

$$
v(p)<1, \quad \forall p \in[0,1], \forall m \geq 2 .
$$

Since the function $v(p)$ is defined and continuous in a compact set, it attains its maximal value. Moreover, (11) implies $\max _{p}(v(p))=K_{m}<1, \forall m \geq 2$, which completes the proof of Lemma 2.3 .

The following assertion, being the main result of this section, deals with the Lebesgue structure of the distribution of the random variable $\xi$.

Theorem 2.1. If either $p=0$ or $p=1$, then the random variable $\xi$ has a degenerate distribution. Otherwise, $\xi$ has a singularly continuous distribution.

Proof. The first statement of the theorem is obvious. Let

$$
M=\prod_{n=1}^{\infty} \max _{i}\left\{\widetilde{p}_{i n}\right\}
$$

It is clear that the latter product diverges to 0 for all $p \in(0,1)$ and that $M=1$ if $p \in\{0,1\}$. According to Lévy's theorem [13, the random variable $\tilde{\xi}$ has either a pure discrete distribution $(p \in\{0,1\})$ or a pure continuous $(p \in(0,1))$ distribution.

The random variable $\tilde{\xi}$, as a random variable with independent $\widetilde{Q}$-symbols, has a pure absolutely continuous distribution if and only if

$$
\prod_{n=1}^{\infty}\left(\sum_{i=0}^{m_{n}-1} \sqrt{q_{i n} \widetilde{p}_{i n}}\right)>0
$$

(see [2]). Since

$$
\sum_{i=0}^{m_{n}-1} \sqrt{q_{i n} \widetilde{p}_{i n}}=\sqrt{\frac{r_{k_{n}}}{r_{k_{n-1}}}} \sum_{i=0}^{l_{n}} \sqrt{C_{l_{n}}^{i} q^{l_{n}-i} p^{i}} \quad \text { and } \quad \frac{r_{k_{n}}}{r_{k_{n-1}}} \leq \frac{1}{l_{n}+1},
$$

we get

$$
\sum_{i=0}^{m_{n}-1} \sqrt{q_{i n} \widetilde{p}_{i n}} \leq \sqrt{\frac{1}{l_{n}+1}} \sum_{i=0}^{l_{n}} \sqrt{C_{l_{n}}^{i} q^{l_{n}-i} p^{i}}
$$

The necessary condition for the convergence of the product

$$
\prod_{n=1}^{\infty}\left(\sum_{i=0}^{l_{n}} \sqrt{q_{i n} \tilde{p}_{i n}}\right)
$$

is given by

$$
\sqrt{\frac{1}{l_{n}+1}} \sum_{i=0}^{l_{n}} \sqrt{C_{l_{n}}^{i} q^{l_{n}-i} p^{i}} \rightarrow 1, \quad n \rightarrow \infty .
$$


If $l_{n}>1$, then Corollary 2.1 and Lemma 2.3 imply that

$$
\sqrt{\frac{1}{l_{n}+1}} \sum_{i=0}^{l_{n}} \sqrt{C_{l_{n}}^{i} q^{l_{n}-i} p^{i}} \leq \max \left\{\max \left\{K_{j}, j=2, \ldots, m_{0}\right\}, K_{0}\right\}<1,
$$

where $m_{0}$ is the number for which the condition

$$
\sqrt{\frac{1}{m+1}} \sum_{i=0}^{m} \sqrt{C_{m}^{i} q^{m-i} p^{i}} \leq K_{0}<1
$$

holds for $m \geq m_{0}$. On the other hand, the inequality $a_{k}<r_{k}$ holds for an infinite number of indices $k$. The latter condition is equaivalent to the condition that the inequality $l_{n}>1$ holds for an infinite number of indices $n$. Therefore the distribution of the random variable $\widetilde{\xi}$ cannot be absolutely continuous. Since the distribution of the random variable $\xi$ is pure, the measure $\mu_{\xi}$ is singular in view of Remark 2.1.

\section{Fractal properties of the Distribution SPECTRUm}

Let $M$ be a fixed bounded subset of a real line. Recall that a family $\Phi_{M}$ of intervals is called a family of locally fine coverings of the set $M$ if, for an arbitrary $\varepsilon>0$, there exists at most a countable $\varepsilon$-covering $\left\{E_{j}\right\}$ of the set $M$ such that $E_{j} \in \Phi_{M}$, that is,

$$
\forall \varepsilon>0 \exists\left\{E_{j}\right\} \text { such that } E_{j} \in \Phi_{M} \text { and }\left|E_{j}\right| \leq \varepsilon \text { for all } j \text { and } M \subset \bigcup_{j} E_{j} .
$$

The Hausdorff $\alpha$-dimensional measure of a subset $E \subset M$ with respect to a given family $\Phi_{M}$ is given by

$$
H^{\alpha}\left(E, \Phi_{M}\right)=\lim _{\varepsilon \rightarrow \infty}\left[\inf _{\left|E_{j}\right| \leq \varepsilon}\left\{\sum_{j}\left|E_{j}\right|^{\alpha}\right\}\right]=\lim _{\varepsilon \rightarrow \infty} m_{\varepsilon}^{\alpha}\left(E, \Phi_{M}\right),
$$

where the infimum is evaluated with respect to all at most countable $\varepsilon$-coverings $\left\{E_{j}\right\}$ of the set $E$ such that $E_{j} \in \Phi_{M}$. The Hausdorff $\alpha$-dimensional measure with respect to a family coincides with the classical Hausdorff $\alpha$-dimensional measure if $M=[0,1]$ and if the infimum is evaluated with respect to the family of all open (closed) intervals. The Hausdorff $\alpha$-dimensional measure of a set is denoted by $H^{\alpha}(E)$.

The nonnegative number

$$
\operatorname{dim}_{H}\left(E, \Phi_{M}\right)=\inf \left\{\alpha: H^{\alpha}\left(E, \Phi_{M}\right)=0\right\}
$$

is called the Hausdorff-Besicovitch dimension of a set $E \subset M$ with respect to a family of locally fine coverings $\Phi_{M}$.

A family $\Phi_{M}$ of locally fine coverings is called faithful if $\operatorname{dim}_{H}\left(E, \Phi_{M}\right)=\operatorname{dim}_{H}(E)$ for all $E \subseteq M$.

Let $\widetilde{\mathcal{A}}_{n}$ be the family of cylindrical intervals of rank $k_{n}$, that is,

$$
\widetilde{\mathcal{A}}_{n}=\left\{E: E=\Delta_{\alpha_{1} \ldots \alpha_{k_{n}}}, \alpha_{i} \in\{0,1\}, i=1,2, \ldots, k_{n}\right\},
$$

and let

$$
\widetilde{\mathcal{A}}=\bigcup_{n=1}^{\infty} \widetilde{\mathcal{A}}_{n}
$$

Lemma 3.1. Let $\sup \left\{l_{n}\right\}<\infty$. Then $\widetilde{\mathcal{A}}$ is a faithful family of coverings on the spectrum, that is,

$$
\operatorname{dim}_{H}(E, \widetilde{\mathcal{A}})=\operatorname{dim}_{H}(E) \quad \text { for all } E \subset S_{\mu} .
$$


Proof. The inequality $\operatorname{dim}_{H}(E, \widetilde{\mathcal{A}}) \geq \operatorname{dim}_{H}(E)$ is obvious, since the family of all subsets of the unit interval contains $\widetilde{\mathcal{A}}$.

Now we are going to prove the converse inequality. Let $\left\{E_{i}\right\}$ be an arbitrary $\varepsilon$-covering of the set $E \subset S_{\mu}$ by intervals $E_{i}=\left(a_{i}, b_{i}\right)$. Since the evaluation of the premeasure $m_{\varepsilon}^{\alpha}(E)$ requires a covering such that $E_{i} \cap E \neq \varnothing$, we assume without loss of generality that $E_{i} \cap E \neq \varnothing$. For an arbitrary $E_{i}$, there exists a cylindrical interval

$$
\Delta_{[n(i)]}:=\Delta_{c_{1} \ldots c_{k_{n(i)}}} \in \widetilde{\mathcal{A}}_{n(i)}
$$

such that $\Delta_{[n(i)]} \subset E_{i}$ and $E_{i}$ does not contain intervals belonging to $\widetilde{\mathcal{A}}_{n(i)-1}$. Any $E_{i}$ cannot contain more than $2 \cdot l_{n(i)}$ cylinders of $\widetilde{\mathcal{A}}_{n(i)}$, since otherwise $E_{i}$ contains a cylindrical interval belonging to $\widetilde{\mathcal{A}}_{n(i)-1}$. Thus the set $E_{i} \cap E$ belongs to a union of at most $2 \cdot l_{n(i)}+2$ isometric cylinders of length $r_{k_{n(i)}}$ belonging to $\widetilde{\mathcal{A}}_{n(i)}$ (and including $\Delta_{[n(i)]}$ ). Hence $\left|\Delta_{[n(i)]}\right|<\varepsilon$ and

$$
\left(2 \cdot l_{n(i)}+2\right)\left|\Delta_{[n(i)]}\right|^{\alpha}<\left(2 \cdot l_{n(i)}+2\right)\left|E_{i}\right|^{\alpha},
$$

whence we conclude that

$$
m_{\varepsilon}^{\alpha}(E, \tilde{\mathcal{A}}) \leq \sum_{i}\left(2 \cdot l_{n(i)}+2\right)\left|\Delta_{[n(i)]}\right|^{\alpha} \leq\left(2 \cdot \sup \left\{l_{n}\right\}+2\right) \sum_{i}\left|E_{i}\right|^{\alpha}
$$

for an arbitrary fixed $\varepsilon>0, \alpha>0$, and for an arbitrary $\varepsilon$-covering of the set $E \subset S_{\mu}$ by intervals $E_{i}$.

Therefore

$$
m_{\varepsilon}^{\alpha}(E, \widetilde{\mathcal{A}}) \leq\left(2 \cdot \sup \left\{l_{n}\right\}+2\right) m_{\varepsilon}^{\alpha}(E) .
$$

Passing to the limit we obtain

$$
H^{\alpha}(E, \widetilde{\mathcal{A}}) \leq\left(2 \cdot \sup \left\{l_{n}\right\}+2\right) H^{\alpha}(E),
$$

whence the desired inequality

$$
\operatorname{dim}_{H}(E, \widetilde{\mathcal{A}}) \leq \operatorname{dim}_{H}(E)
$$

follows. Lemma 3.1 is proved.

Using the latter lemma we evaluate the Hausdorff-Besicovitch dimension of the topological support $S_{\mu}$ (that is, the dimension of the minimal closed support of the measure $\mu$ ).

Theorem 3.1. Let $\sup \left\{l_{n}\right\}<\infty$. Then the Hausdorff-Besicovitch dimension of the topological support $S_{\mu}$ is equal to

$$
\operatorname{dim}_{H}\left(S_{\mu}\right)=\varliminf_{n \rightarrow \infty}\left(\frac{\sum_{j=1}^{n} \ln \left(l_{j}+1\right)}{-\ln r_{k_{n}}}\right) .
$$

Proof. 1) First we prove that

$$
\operatorname{dim}_{H}\left(S_{\mu}\right) \leq \varliminf_{n \rightarrow \infty}\left(\frac{\sum_{j=1}^{n} \ln \left(l_{j}+1\right)}{-\ln r_{k_{n}}}\right) .
$$

The spectrum $S_{\mu}$ can be covered by $\prod_{j=1}^{n}\left(l_{j}+1\right)$ cylindrical intervals that belong to $\widetilde{\mathcal{A}}$ and whose diameter is equal to $r_{k_{n}}$. Thus

$$
m_{r_{k_{n}}}^{\alpha}\left(S_{\mu}\right) \leq r_{k_{n}}^{\alpha} \cdot \prod_{j=1}^{n}\left(l_{j}+1\right) .
$$


If

$$
\varliminf_{n \rightarrow \infty} r_{k_{n}}^{\alpha} \cdot \prod_{j=1}^{n}\left(l_{j}+1\right)<1
$$

then $H^{\alpha}\left(S_{\mu}\right) \leq 1$. It is easy to check that (13) follows from

$$
\alpha \geq \underset{n \rightarrow \infty}{\lim _{n \rightarrow \infty}}\left(\frac{\sum_{j=1}^{n} \ln \left(l_{j}+1\right)}{-\ln r_{k_{n}}}\right)=: a .
$$

Hence $H^{\alpha}\left(S_{\mu}\right)=0$ for all $\alpha>a$ and, as a result, $\operatorname{dim}_{H}\left(S_{\mu}\right) \leq a$.

2) Next we prove that

$$
\operatorname{dim}_{H}\left(S_{\mu}\right) \geq \underset{n \rightarrow \infty}{\lim _{n \rightarrow \infty}}\left(\frac{\sum_{j=1}^{n} \ln \left(l_{j}+1\right)}{-\ln r_{k_{n}}}\right) .
$$

The spectrum $S_{\mu}$ of a generalized infinite Bernoulli convolution does not depend on the choice of a matrix $P=\left\|p_{i k}\right\|, p_{0 k} \in(0,1)$. Consider an auxiliary probability measure $\mu^{T}$ with independent $\widetilde{Q}$-symbols whose spectrum coincides with the spectrum of the random variable under consideration. Now we choose a matrix $P=\left\|\widetilde{p}_{i k}\right\|$ for which the corresponding measure $\mu^{T}$ is "uniformly distributed" in the topological support, that is, the $\mu^{T}$-measure of every cylindrical interval belonging to $\widetilde{\mathcal{A}}_{n}$ is equal to

$$
\left(\prod_{j=1}^{n}\left(l_{j}+1\right)\right)^{-1}
$$

For a given $\varepsilon>0$, let $\left\{V_{i}\right\}$ be an $\varepsilon$-covering of the set $S_{\mu}$ by cylindrical intervals belonging to $\widetilde{\mathcal{A}}_{n(i)}, r_{k_{n(i)}} \leq \varepsilon$, for all $i \in \mathbf{N}$. Then

$$
\begin{aligned}
1 & =\mu^{T}\left(S_{\mu}\right)=\mu^{T}\left(\bigcup_{i} V_{i}\right) \leq \sum_{i} \mu^{T}\left(V_{i}\right)=\sum_{i} \frac{1}{\prod_{j=1}^{n(i)}\left(l_{j}+1\right)} \\
& =\sum_{i}\left|V_{i}\right|^{\log _{\left|V_{i}\right|}\left(\frac{1}{\prod_{j=1}^{n(i)}\left(l_{j}+1\right)}\right)}=\sum_{i}\left|V_{i}\right|^{-\log _{r_{k}}\left(\prod_{j=1}^{n(i)}\left(l_{j}+1\right)\right)} .
\end{aligned}
$$

The definition of the number $a$ implies that, for an arbitrary $\delta>0$, there exists $n_{0} \in \mathbf{N}$ such that

$$
\frac{\sum_{j=1}^{n} \ln \left(l_{j}+1\right)}{-\ln r_{k_{n}}} \geq a-\delta
$$

for all $n>n_{0}$. We choose $n^{*}$ such that $n^{*}>n_{0}$ and $r_{k_{n^{*}}} \leq \varepsilon$. Thus

$$
1=\mu^{T}\left(S_{\mu}\right) \leq \sum_{i}\left|V_{i}\right|^{a-\delta}
$$

for an arbitrary $r_{k_{n^{*}}}$-covering of the spectrum $S_{\mu}$ by cylindrical intervals belonging to $\widetilde{\mathcal{A}}$.

Hence

$$
1 \leq m_{r_{n^{*}}}^{a-\delta}\left(S_{\mu}, \widetilde{\mathcal{A}}\right) \leq H^{a-\delta}\left(S_{\mu}, \widetilde{\mathcal{A}}\right) \quad \text { for all } \delta>0
$$

and $H^{\alpha}\left(S_{\mu}, \widetilde{\mathcal{A}}\right) \geq 1$ for all $\alpha<a$. The preceding lemma implies that

$$
\operatorname{dim}_{H}\left(S_{\mu}\right)=\operatorname{dim}_{H}\left(S_{\mu}, \widetilde{\mathcal{A}}\right) \geq \underline{\underline{\lim }}\left(\frac{\sum_{j=1}^{n} \ln \left(l_{j}+1\right)}{-\ln r_{k_{n}}}\right) .
$$


Now we show that all probability measures of the class under consideration belong to the so-called infinite Bernoulli convolutions with essential intersections. First we evaluate the Hausdorff-Besicovitch dimension of the set of those points for which there are continuum many different representations and the dimension of the set of points that have a finite number of representations.

Theorem 3.2. Let $\sup \left\{l_{n}\right\}<\infty$. Then

1) the Hausdorff-Besicovitch dimension of the set of points that have a finite number of representations (5) is equal to

$$
\varliminf_{n \rightarrow \infty}\left(\frac{n \ln 2}{-\ln r_{k_{n}}}\right)
$$

2) the Hausdorff-Besicovitch dimension of the set of points that have continuum many representations (5) is equal to

$$
\varliminf_{n \rightarrow \infty}\left(\frac{\sum_{j=1}^{n} \ln \left(l_{j}+1\right)}{-\ln r_{k_{n}}}\right) .
$$

Proof. We recall some main properties of the Hausdorff-Besicovitch dimension (see [9] for details):

B1) if $E_{1} \subset E_{2}$, then $\operatorname{dim}_{H}\left(E_{1}\right) \leq \operatorname{dim}_{H}\left(E_{2}\right)$;

B2) $\operatorname{dim}_{H}\left(\bigcup_{n} E_{n}\right)=\sup _{n} \operatorname{dim}_{H}\left(E_{n}\right)$;

B3) if $E_{1}$ and $E_{2}$ are homothetic, then $\operatorname{dim}_{H}\left(E_{1}\right)=\operatorname{dim}_{H}\left(E_{2}\right)$.

Given a series $\sum_{k=1}^{\infty} a_{k}$, we follow the procedure described in Section2 for constructing the numerial sequences $\left\{l_{n}\right\}$ and $\left\{m_{n}\right\}$ and the corresponding sequence of sets $\left\{B_{n}\right\}$. We construct an auxiliary sequence of sets $\left\{L_{j}\right\}$ such that

$$
\begin{aligned}
L_{j}:=\{x: & \tilde{\Delta}_{\gamma_{1} \gamma_{2} \ldots \gamma_{n} \ldots}, \gamma_{n} \in B_{n}, \text { if } n \in\{1,2, \ldots, j-1\}, \\
& \text { and } \left.\gamma_{n} \in\left\{0, m_{n}-1\right\}, \text { if } n \in \mathbf{N} \backslash\{1,2, \ldots, j-1\}\right\} .
\end{aligned}
$$

The set $L_{1}$ coincides with the set of those points that have a unique representation (5) except a countable set of points. The reasoning similar to that used in the proof of Theorem 3.1 shows that

$$
\operatorname{dim}_{H}\left(L_{1}\right)=\varliminf_{n \rightarrow \infty}\left(\frac{n \ln 2}{-\ln r_{k_{n}}}\right) .
$$

Now we are going to show that $\operatorname{dim}_{H}\left(L_{j}\right)=\operatorname{dim}_{H}\left(L_{1}\right)$ for all $j \in \mathbf{N}$. Indeed, let $j \in \mathbf{N} \backslash\{1\}$. Then

$$
L_{1}=\bigcup_{i}^{2^{j-1}} L_{1}^{(i)}
$$

where $\left\{L_{1}^{(i)}\right\}_{i=1, \ldots, 2^{j-1}}$ are isometric sets whose intersection consists of at most one point and where

$$
\begin{aligned}
L_{1}^{(1)}:=\{x: x & =\tilde{\Delta}_{\gamma_{1} \gamma_{2} \ldots \gamma_{n} \ldots}, \gamma_{n}=0 \text { if } n \in\{1,2, \ldots, j-1\}, \\
& \text { and } \left.\gamma_{n} \in\left\{0, m_{n}-1\right\} \text { if } n \in \mathbf{N} \backslash\{1,2, \ldots, j-1\}\right\} .
\end{aligned}
$$

The equality

$$
\operatorname{dim}_{H}\left(L_{1}^{(1)}\right)=\operatorname{dim}_{H}\left(L_{1}\right)
$$


follows from properties B2) and B3). The set $L_{j}$ can be represented in the form

$$
L_{j}=\bigcup_{t=1}^{\prod_{i=1}^{j-1}\left(l_{i}+1\right)} L_{j}^{(t)}
$$

where $\left\{L_{j}^{(t)}\right\}_{t=1, \ldots, \prod_{i=1}^{j-1}\left(l_{i}+1\right)}$ are isometric sets whose intersection contains at most one point, and $L_{1}^{(1)} \in\left\{L_{j}^{(t)}\right\}_{t=1, \ldots, \prod_{i=1}^{j-1}\left(l_{i}+1\right)}$. Then properties B2) and B3) together with equality (14) imply that

$$
\operatorname{dim}_{H}\left(L_{j}\right)=\operatorname{dim}_{H}\left(L_{1}\right) .
$$

The preceding equality together with B2) yields

$$
\operatorname{dim}_{H}\left(\bigcup_{i} L_{i}\right)=\sup _{i} \operatorname{dim}_{H}\left(L_{i}\right)=\operatorname{dim}_{H}\left(L_{1}\right) .
$$

The set of points that have a finite number of representations in the form of (5) coincides with the set $\bigcup_{j=1}^{\infty} L_{j}$. This completes the proof of the first statement of Theorem 3.2

Let $L^{*}$ be the set of points for which there exist continuum many different representations in the form of (5). If

$$
\operatorname{dim}_{H} S_{\mu}>\operatorname{dim}_{H} L_{1}=\underset{n \rightarrow \infty}{\lim }\left(\frac{n \ln 2}{-\ln r_{k_{n}}}\right),
$$

then the equality $S_{\mu}=L^{*} \cup\left(\bigcup_{j=1}^{\infty} L_{j}\right)$ and property $B 2$ imply that $\operatorname{dim}_{H} L^{*}=\operatorname{dim}_{H} S_{\mu}$. Hence almost all points (in the sense of the Hausdorff-Besicovitch dimension) of the spectrum $S_{\mu}$ have continuum many different representations in the form of (5).

Now we show that $\operatorname{dim}_{H} L^{*}=\operatorname{dim}_{H} S_{\mu}$, even in the case where

$$
\varliminf_{n \rightarrow \infty}\left(\frac{\sum_{j=1}^{n} \ln \left(l_{j}+1\right)}{-\ln r_{k_{n}}}\right)=\varliminf_{n \rightarrow \infty}\left(\frac{n \ln 2}{-\ln r_{k_{n}}}\right),
$$

that is where $\operatorname{dim}_{H} S_{\mu}=\operatorname{dim}_{H} L_{1}$ (this is the case, in particular, if $l_{n}=1, n \neq 2^{s}$, or $l_{n}=2, n=2^{s}$ ).

Since $l_{n}>1$ for infinitely many indices $n$, one can choose a sufficiently "sparse" subsequence $n_{t}$ in such a way that the sets $B_{n_{t}}$ contain at least three elements. In each of the sets $B_{n_{t}} \backslash\left\{0, m_{n_{t}}-1\right\}$, we choose an arbitrary element and denote it by $\theta_{t}$. Consider the set

$$
\begin{aligned}
K_{1}=\left\{x: x:=\tilde{\Delta}_{\gamma_{1} \gamma_{2} \ldots \gamma_{n} \ldots}, \text { where } \gamma_{k} \in\left\{0, m_{k}-1\right\}\right. & \text { for } k \notin\left\{n_{t}\right\} \\
& \text { and } \left.\gamma_{n_{t}}=\theta_{t} \text { for all } t \in \mathbf{N}\right\} .
\end{aligned}
$$

Each point of the set $K_{1}$ has continuum many representations of the form (5), that is, $K_{1} \subset L^{*}$. The reasoning being similar to that used in thee proof of Theorem 3.1 proves that

$$
\operatorname{dim}_{H}\left(K_{1}\right)=\varliminf_{n \rightarrow \infty}\left(\frac{\sum_{i=1}^{n} \ln \left(z_{i}+1\right)}{-\ln r_{k_{n}}}\right),
$$

where $z_{i}=0$ for $i \in\left\{n_{t}\right\}$, and $z_{i}=1$ for $i \notin\left\{n_{t}\right\}$. Then

$$
\operatorname{dim}_{H}\left(K_{1}\right)=\varliminf_{n \rightarrow \infty}\left(\frac{(n-\tau(n)) \ln 2}{-\ln r_{k_{n}}}\right)=\varliminf_{n \rightarrow \infty}\left(\frac{(n-\tau(n))}{n} \frac{n \ln 2}{-\ln r_{k_{n}}}\right),
$$

where $\tau(n)$ is the number of members of the sequence $\left\{n_{t}\right\}$ being less than $n$. Since the sequence $\left\{n_{t}\right\}$ is sufficiently "sparse" in the sense that $\tau(n) / n \rightarrow 0(n \rightarrow \infty)$, we have $\operatorname{dim}_{H} K_{1}=\operatorname{dim}_{H} L_{1}$. Hence $\operatorname{dim}_{H} L^{*}=\operatorname{dim}_{H} L_{1}$. 
Corollary 3.1. If $\lambda\left(S_{\mu}\right)>0$, then almost all (in the sense of the Lebesgue measure) points of the spectrum have continuum many different representations (5).

\section{Fine fractal properties of the Distribution OF THE RANDOM VARIABLE $\xi$}

The spectrum is a rather rough characteristic of a singularly continuous probability distribution, since even an uncountable family of mutually singular distributions may have a joint spectrum. As an example, one can consider the class of infinite Bernoulli convolutions of the form $\xi=\sum_{k=1}^{\infty} \xi_{k} / 2^{k}$, where $\xi_{k}$ are independent random variables assuming the values 0 and 1 with probabilities $p \in\left(0, \frac{1}{2}\right)$ and $1-p$, respectively. Thus the Hausdorff-Besicovitch dimension of the spectrum of a measure is a too rough fractal characteristic of a singular measure as well. The Hausdorff dimension of a distribution much better characterizes the properties of a singular distribution.

Recall that the number

$$
\operatorname{dim}_{H}(\tau)=\inf \left\{\operatorname{dim}_{H}(E), E \in \mathcal{B}_{\tau}\right\}
$$

is called the Hausdorff dimension of the distribution of a random variable $\tau$, where $\mathcal{B}_{\tau}$ is the class of all Borel supports $\left(\mathcal{B}_{\tau}\right.$ needs not necessarily be closed) of the random variable $\tau$; that is,

$$
\mathcal{B}_{\tau}=\left\{E: E \in \mathcal{B}, \mathrm{P}_{\tau}(E)=1\right\} .
$$

Let $v$ be a continuous probability measure defined on Borel sets of the interval $[0,1]$, $E_{0}$ a certain fixed subset of the unit interval, and $\Phi\left(E_{0}\right)$ a family of intervals belonging to $[0,1]$ such that, for $E_{0}$ and for an arbitrary $\varepsilon>0$, there exists at most countable $(v-\varepsilon)$-covering $\left\{E_{j}\right\}$ of the set $E_{0}$ with $E_{j} \in \Phi\left(E_{0}\right)$ and $v\left(E_{j}\right) \leq \varepsilon$. Then the Hausdorff $(v-\alpha)$-measure of an arbitrary set $E \subset E_{0}$ is defined as follows:

$$
H^{\alpha}\left(E, v, \Phi\left(E_{0}\right)\right)=\lim _{\varepsilon \rightarrow \infty}\left[\inf _{v\left(E_{j}\right) \leq \varepsilon}\left\{\sum_{j} v^{\alpha}\left(E_{j}\right)\right\}\right]=\lim _{\varepsilon \rightarrow \infty} m_{\varepsilon}^{\alpha}(E, v, \Phi),
$$

where $E_{j} \in \Phi\left(E_{0}\right)$ and $\bigcup_{j} E_{j} \supset E$.

The number

$$
\operatorname{dim}_{v}\left(E, \Phi\left(E_{0}\right)\right)=\inf \left\{\alpha: H^{\alpha}\left(E, v, \Phi\left(E_{0}\right)\right)=0\right\}
$$

is called the Hausdorff-Billingsley dimension of the set $E$ with respect to the measure $v$ and family of coverings $\Phi\left(E_{0}\right)$.

Theorem 4.1. Let $\sup \left\{l_{n}\right\}<\infty$. Then the Hausdorff dimension of the distribution of the random variable $\xi$ is equal to

$$
\operatorname{dim}_{H}\left(\mu_{\xi}\right)=\lim _{n \rightarrow \infty} \frac{\sum_{j=1}^{n} h_{j}}{-\ln r_{k_{n}}}
$$

where

$$
h_{j}=-\sum_{i=0}^{l_{j}} \tilde{p}_{i j} \ln \tilde{p}_{i j} .
$$

Proof. Note that the evaluation of the Hausdorff dimension of the random variable $\xi$ can be restricted to the case of supports being subsets of the spectrum of $\xi$.

Let $\widetilde{\Delta}_{[n]}(x):=\widetilde{\Delta}_{a_{1}(x) a_{2}(x) \ldots a_{n}(x)}$ be a $\widetilde{Q}$-cylindrical interval of rank $n$ that contains a point $x$ of the spectrum $S_{\xi}$. Note that the class of all cylindrical intervals coincides with $\widetilde{\mathcal{A}}$ (see (12) ). Let $\mu$ be the probability measure corresponding to the distribution of the random variable $\xi$, that is,

$$
\mu(E)=\mathrm{P}\{\xi \in E\}
$$


for all $E \in \mathcal{B}$. Let $\lambda$ denote the Lebesgue measure in $[0,1]$. Then

$$
\begin{gathered}
\mu\left(\widetilde{\Delta}_{[n]}(x)\right)=\tilde{p}_{a_{1}(x) 1} \cdot \tilde{p}_{a_{2}(x) 2} \cdot \ldots \cdot \tilde{p}_{a_{n}(x) n}, \\
\lambda\left(\widetilde{\Delta}_{[n]}(x)\right)=q_{a_{1}(x) 1} \cdot q_{a_{2}(x) 2} \cdot \ldots \cdot q_{a_{n}(x) n}=r_{k_{n}} .
\end{gathered}
$$

Consider

$$
\frac{\ln \mu\left(\widetilde{\Delta}_{[n]}(x)\right)}{\ln \lambda\left(\widetilde{\Delta}_{[n]}(x)\right)}=\frac{\sum_{j=1}^{n} \ln \tilde{p}_{a_{j}(x) j}}{\ln r_{k_{n}}}
$$

If $x=\widetilde{\Delta}_{a_{1}(x) a_{2}(x) \ldots a_{n}(x) \ldots}$ is chosen randomly such that $\mathrm{P}\left(a_{j}(x)=i\right)=\tilde{p}_{i j}$ (in other words, the distribution of the random variable $x$ corresponds to the measure $\mu$ ), then

$$
\left\{\eta_{j}\right\}=\left\{\eta_{j}(x)\right\}:=\left\{\ln \tilde{p}_{a_{j}(x) j}\right\}
$$

is a sequence of independent random variables with the following distributions:

$$
\mathrm{P}\left\{\eta_{j}=\ln \tilde{p}_{i j}\right\}=\tilde{p}_{i j}, \quad i=0, \ldots, m_{j}-1 .
$$

It is also clear that

$$
\begin{gathered}
\mathrm{E} \eta_{j}=\sum_{i=0}^{m_{j}-1} \tilde{p}_{i j} \ln \tilde{p}_{i j}=-h_{j}, \quad\left|h_{j}\right| \leq c_{1}<\infty \\
\mathrm{E} \eta_{j}^{2}=\sum_{i=0}^{m_{j}-1} \tilde{p}_{i j} \ln ^{2} \tilde{p}_{i j} \leq c_{2}<\infty .
\end{gathered}
$$

Moreover, the constants $c_{1}$ and $c_{2}$ do not depend on $j$, since the functions $\nu(x)=x \ln x$ and $\varphi(x)=x \ln ^{2} x$ are bounded in the interval $[0,1]$ and since $\sup l_{j}<\infty$.

Put

$$
S_{n}:=\eta_{1}(x)+\eta_{2}(x)+\cdots+\eta_{n}(x) .
$$

By the Kolmogorov strong law of large numbers,

$$
\lim _{n \rightarrow \infty} \frac{\left(S_{n}-\mathrm{E}\left(S_{n}\right)\right)}{n}=0
$$

for $\mu$-almost all points $x \in[0,1]$. Note that

$$
\mathrm{E}\left(S_{n}\right)=\mathrm{E}\left(\eta_{1}\right)+\mathrm{E}\left(\eta_{2}\right)+\cdots+\mathrm{E}\left(\eta_{n}\right)=-\left(h_{1}+h_{2}+\cdots+h_{n}\right) .
$$

Put $H_{n}:=h_{1}+h_{2}+\cdots+h_{n}, D=\underline{\lim }_{n \rightarrow \infty} H_{n} /\left(-\ln r_{k_{n}}\right)$ and consider the set

$$
T=\left\{x: \lim _{n \rightarrow \infty}\left(\frac{S_{n}}{\ln r_{k_{n}}}-\frac{H_{n}}{-\ln r_{k_{n}}}\right)=0\right\}=\left\{x: \lim _{n \rightarrow \infty}\left(\frac{\frac{1}{n}\left(S_{n}-\mathrm{E}\left(S_{n}\right)\right)}{\frac{1}{n} \ln r_{k_{n}}}\right)=0\right\} .
$$

Since $r_{k_{n}} \leq \frac{1}{2^{n}}$, the product $\frac{1}{n} \ln r_{k_{n}}$ is separated from zero. Thus $\mu(T)=1$, whence $\operatorname{dim}_{\mu}(T, \widetilde{\mathcal{A}})=1$. Let

$$
\begin{aligned}
& T_{1}=\left\{x: \underline{\lim }\left(\frac{S_{n}}{\ln r_{k_{n}}}-\frac{H_{n}}{-\ln r_{k_{n}}}\right)=0\right\}, \\
& T_{2}=\left\{x: \underline{\lim _{n \rightarrow \infty}} \frac{S_{n}}{\ln r_{k_{n}}} \leq \underline{\lim } \frac{H_{n}}{-\ln r_{k_{n}}}\right\} \\
&=\left\{x: \underline{\lim _{n \rightarrow \infty}} \frac{\ln \mu\left(\widetilde{\Delta}_{[n]}(x)\right)}{\ln \lambda\left(\widetilde{\Delta}_{[n]}(x)\right)} \leq \underline{\lim } \frac{H_{n}}{-\ln r_{k_{n}}}\right\}, \\
& T_{3}=\left\{x: \underline{\lim _{n \rightarrow \infty}} \frac{S_{n}}{\ln r_{k_{n}}} \geq \underline{\underline{\lim }} \frac{H_{n}}{-\ln r_{k_{n}}}\right\} \\
&=\left\{x: \underset{n \rightarrow \infty}{\lim } \frac{\ln \mu\left(\widetilde{\Delta}_{[n]}(x)\right)}{\ln \lambda\left(\widetilde{\Delta}_{[n]}(x)\right)} \geq \underline{\underline{\lim }} \frac{H_{n}}{-\ln r_{k_{n}}}\right\} .
\end{aligned}
$$


It is obvious that $T \subset T_{1}$. Similarly to the proof of Theorem 1 in paper 4], one can prove that $T_{1} \subset T_{3}$ and $T \subset T_{2}$.

By Theorem 2.1 of $[6], \operatorname{dim}_{\lambda}\left(T_{2}, \widetilde{\mathcal{A}}\right) \leq D$. Taking into account the inclusion $T \subset T_{2}$ we obtain

$$
\operatorname{dim}_{\lambda}(T, \widetilde{\mathcal{A}}) \leq D
$$

Since

Theorem 2.2 of [6] implies

$$
T \subset T_{3}=\left\{x: \varliminf_{n \rightarrow \infty} \frac{\ln \mu\left(\widetilde{\Delta}_{[n]}(x)\right)}{\ln \lambda\left(\widetilde{\Delta}_{[n]}(x)\right)} \geq D\right\},
$$

$$
\operatorname{dim}_{\lambda}(T, \widetilde{\mathcal{A}}) \geq D \cdot \operatorname{dim}_{\mu}(T, \widetilde{\mathcal{A}})=D \cdot 1=D .
$$

Thus $\operatorname{dim}_{\lambda}(T, \widetilde{\mathcal{A}})=D$. Since $\lambda$ is the Lebesgue measure in the interval $[0,1]$, we get

$$
\operatorname{dim}_{H}(T, \widetilde{\mathcal{A}})=\operatorname{dim}_{\lambda}(T, \widetilde{\mathcal{A}})=D .
$$

By Lemma 3.1, $\operatorname{dim}_{H}(T, \widetilde{\mathcal{A}})=\operatorname{dim}_{H}(T)$, whence $\operatorname{dim}_{H}(T)=D$.

Next we prove that the set $T$ constructed above is the minimal probability support of the measure $\mu$. Let $C$ be a support of the measure $\mu$, that is, $\mu(C)=1$. It is clear that $C_{1}=C \cap T$ is also a support of the measure $\mu$ and $C_{1} \subset C$. Therefore $\operatorname{dim}_{H}\left(C_{1}\right) \leq \operatorname{dim}_{H}(C)$ and $C_{1} \subset T$.

Then we show that $\operatorname{dim}_{H}\left(C_{1}\right)=\operatorname{dim}_{H}(T)$. Since $C_{1} \subset T$, we conclude that

$$
\operatorname{dim}_{H}\left(C_{1}\right) \leq \alpha_{0}(T)=D .
$$

On the other hand,

$$
C_{1} \subset T \subset T_{3}=\left\{x: \varliminf_{n \rightarrow \infty} \frac{\ln \mu\left(\widetilde{\Delta}_{[n]}(x)\right)}{\ln \lambda\left(\widetilde{\Delta}_{[n]}(x)\right)} \geq D\right\} .
$$

Thus Theorems 2.1 and 2.2 of [6] together with Lemma 3.1 imply that

$$
\operatorname{dim}_{H}\left(C_{1}\right)=\operatorname{dim}_{\lambda}\left(C_{1}, \widetilde{\mathcal{A}}\right) \geq D \cdot \operatorname{dim}_{\mu}(T, \widetilde{\mathcal{A}}) \geq D \cdot \operatorname{dim}_{\mu}\left(C_{1}, \widetilde{\mathcal{A}}\right)=D \cdot 1=D .
$$

Corollary 4.1. The random variable $\xi$ may have a superfractal $\left(\operatorname{dim}_{H}\left(\mu_{\xi}\right)=1\right)$ singular distribution even if the random variables $\xi_{k}$ are identically distributed. This property holds, in particular, if $p=q=\frac{1}{2}, a_{k_{n}}=r_{k_{n}}$ for all $n \in \mathbf{N}$, and

$$
l_{n}=\left\{\begin{array}{ll}
1, & \text { if } n \neq 2^{s}, \\
2, & \text { if } n=2^{s},
\end{array} \quad s \in \mathbf{N} .\right.
$$

\section{BIBLIOGRAPHY}

1. S. Albeverio and G. Torbin, On fine fractal properties of generalized infinite Bernoulli convolutions, Bull. Sci. Math. 132 (2008), no. 8, 711-727. MR2474489 (2010e:28006)

2. S. Albeverio, V. Koshmanenko, M. Pratsiovytyi, and G. Torbin, On fine structure of singularly continuous probability measures and random variables with independent $\widetilde{Q}$-symbols, Methods Funct. Anal. Topology 17 (2011), no. 2, 97-111. MR2849470 (2012g:60006)

3. S. Albeverio and G. Torbin, Image measures of infinite product measures and generalized Bernoulli convolutions, Proceedings of Dragomanov National Pedagogical University, ser. 1, Physics and Mathematics 5 (2004), 248-264.

4. S. Albeverio and G. Torbin, Fractal properties of singularly continuous probability distributions with independent $Q^{*}$-digits, Bull. Sci. Math. 129 (2005), no. 4, 356-367. MR2134126 (2006b:28013)

5. J. C. Alexander and D. Zagier, The entropy of a certain infinitely convolved Bernoulli measure, J. London Math. Soc. 44 (1991), 121-134. MR1122974 (92g:28035)

6. P. Billingsley, Hausdorff dimension in probability theory II, Ill. J. Math. 5 (1961), 291-198. MR0120339 (22:11094) 
7. M. Cooper, Dimension, measure and infinite Bernoulli convolutions, Math. Proc. Cambr. Phil. Soc. 124 (1998), 135-149. MR1620520 (99h:28013)

8. P. Erdős, On a family of symmetric Bernoulli convolutions, Amer. J. Math. 61 (1939), 974-975. MR0000311 (1:52a)

9. K. J. Falconer, Fractal Geometry, John Wiley \& Sons, 1990. MR1102677 (92j:28008)

10. A. M. Garsia, Arithmetic properties of Bernoulli convolutions, Trans. Amer. Math. Soc. 102 (1962), 409-432. MR0137961 (25:1409)

11. Ya. Gontcharenko, M. Pratsiovytyi, and G. Torbin, On fractal properties of some Bernoulli convolutions, Prob. Theory and Math. Statist. 79 (2009), 39-55. MR2494534 (2010d:60101)

12. B. Jessen and A. Wintner, Distribution function and Riemann Zeta-function, Trans. Amer. Math. Soc. 38 (1935), 48-88. MR.1501802

13. P. Lévy, Sur les séries dont les termes sont des variables indépendantes, Studia Math. 3 (1931), $119-155$.

14. Y. Peres, W. Schlag, and B. Solomyak, Sixty years of Bernoulli convolutions, Fractal Geometry and Stochastics II, Progress in Probab., vol. 46, 2000, pp. 39-65. MR1785620 (2001m:42020)

15. Y. Peres and B. Solomyak, Absolute continuity of Bernoulli convolutions, a simple proof, Math. Res. Lett. 3 (1996), no. 2, 231-239. MR.1386842 (97f:28006)

16. B. Solomyak, On the random series $\sum \pm \lambda^{n}$ (an Erdös problem), Ann. Math. 142 (1995), 611625. MR.1356783 (97d:11125)

17. M. V. Pratsevytyı̌ and G. M. Torbin, A certain class of Jessen-Wintner type random variables, Dopov. Nats. Akad. Nauk Ukr. Mat. Prirodozn. Tekh. Nauki (1998), no. 4, 48-54. (Ukrainian)

18. M. V. Pratsevytyı̆, Fractal Approach in Studies of Singular Distributions, Dragomanov National Pedagogical University Publishing House, Kyiv, 1998. (Ukrainian)

19. G. M. Torbin, Multifractal analysis of singularly continuous probability measures, Ukrain. Mat. Zh. 57 (2005), no. 5, 837-857; English transl. in Ukrainian Math. J. 57 (2005), no. 5, 837-857. MR2209816 (2007f:28010)

Department of Mathematical Analysis and Differential Equations, Dragomanov National Pedagogical University, Pirogov Street, 9, Kyiv 01130, Ukraine

E-mail address: mykola.lebid@gmail.com

Department of Mathematical Analysis and Differential Equations, Dragomanov National Pedagogical University, Pirogov Street, 9, Kyiv 01130, Ukraine

E-mail address: torbin7@gmail.com, torbin@iam.uni-bonn.de

Received 09/APR/2012

Translated by N. SEMENOV 